\title{
Information Processing Analysis of the Chevreul Pendulum Illusion
}

\author{
Randolph D. Easton \\ Boston College
}

\author{
Ronald E. Shor \\ University of New Hampshire
}

\begin{abstract}
An information processing investigation was performed to quantify the Chevreul pendulum effect: the tendency of a small pendulum, when suspended from the hand and imaginatively concentrated on, to oscillate seemingly of its own accord. Using a time exposure photographic measurement technique, electronically automated visual and auditory imaginal prompts were presented to the subject during imaginal processing tasks. It was found that the pendulum effect was enhanced when vision of actual pendulum oscillations was permitted and visual or auditory spatially oscillating stimuli were present. Visual spatially oscillating stimuli were superior to their auditory counterparts. Results were discussed in terms of ideomotor and visual capture interpretations of signal and imaginal processing.
\end{abstract}

The construct of ideomotor behavior-the tendency of thoughts and images of action to become actualized in behavior automatically - was first brought into the boundaries of scientific psychology in a series of experiments on alleged spiritualistic phenomena by the French chemist Michel Chevreul (1833, 1854). Later in the century James (1890) succinctly stated the issue by proposing that the perceptual image or idea of action initiates performance of that action when no other contradictory idea is present in the mind. The psychological concept of an idea, however, proved not to be respectable within rigorous behavior theory and the ideomotor principle eventually fell into disrepute (Thorndike, 1913; Watson, 1930).

Recent lines of research, however, suggest the efficacy of the proposed ideomotor principle as a means of exploring certain cognitive processes. Greenwald and his associates (Greenwald, 1970, 1972; Greenwald \& Shulman, 1973) have demonstrated the utility of the ideomotor principle as an

This study was supported in part by U. S. National Defense Education Act Title IV Fellowship 70-02501 awarded to the first author and by Departmental Science Development Grant GU3845 from the National Science Foundation. We wish to thank R. I. Erickson, G. A. Forsyth, and J. E. Limber for their helpful comments.

Requests for reprints should be sent to Randolph D. Easton, Psychology Department, Boston College, Chestnut Hill, Massachusetts 02167. interpretation of the manner in which external stimulation and sensory feedback may be involved in the regulation of skilled performance. In other endeavors a renewed interest in mental imagery has resulted in the development of reliable empirical methods to investigate nonverbal representational processes (Sheehan, 1972). Finally, modern electromyographic techniques have provided substantial evidence confirming the ideomotor correlations obtained by early behaviorists (McGuigan \& Schoonover, 1973). Whether or not a motor theory of consciousness has validity (Miller, 1969; Smith, K., 1964, 1969; Smith, S. M., Brown, Teman, \& Goodman, 1947), it is evident that experiments designed to explore the topography of covert muscle movements that are correlated with the symbolic processes should help elucidate the nature of imaginal representation. This article reports the development of a method aimed at exploring the relations among covert muscle behavior, mental imagery, and the perception of external stimulation.

The present method uses a pendulum-type device similar to that used by Chevreul which consists of a weighted body suspended by a cord from the fingertips. The pendulum was found to oscillate back and forth when imaginatively concentrated on, seemingly of its own accord. The actual experience of seeing the pendulum move is an 
illusion since an operator does not feel his muscles initiate and maintain the movements, when in fact they are the sole agency. Theoretically, the illusion could arise in one of two ways that are not necessarily exclusive alternatives. First, the movement could indeed occur in an ideomotor way, as an automatic motoric consequence of a central neural mechanism's activity, that is, of a kinetic representation or image. Alternatively (or in addition), the subject, in an effort to produce what he has been instructed to do, namely, to construct a visual imagination of movement, may initiate pendular movements via small hand movements of which he is unaware. The literature on visual capture (Gibson, 1933; Nielsen, 1963; Pick, Warren, \& Hay, 1969; Rock, 1966; Ryan, 1940) indicates that conscious monitoring of kinesthetic information is difficult in the presence of simultaneous visual information. Thus, without violating the general instruction not to consciously or deliberately move the pendulum, the subject may do so in an effort to create a kinetic image. Such an effect would be quite consistent with the Perky phenomenon which indicates that low intensity stimuli may be confused with an image and that partially processed sensory input could trigger a seemingly autonomous image (Perky, 1910; Segal, 1972).

The first question asked in previous experiments designed to quantify the pendulum effect was whether the pendulum moves simply because the hand cannot be held absolutely still. A conceptual analysis of the physical principles of the pendulum revealed that all excitatory movements not in or near the resonant frequency of the pendulum would lessen the resonant energy of the system (Crandall \& Marks, 1963). As only a small portion of natural bodily movements would be in phase with the period of the pendulum, the overall effect of random movements would be to attenuate the resonant energy. The periodicity of covert responses correlated with subjects' imagined pendular movement, however, should be close to the resonant frequency of the pendulum, allowing it to act as a mechanical amplifier. A very simple initial experiment was designed to substantiate this argument empirically. A photographic time exposure technique (described below) was used to compare the magnitude of pendulum oscillations when subjects imagined the pendulum moved back and forth, imagined the pendulum remained absolutely still, or maintained as neutral an attitude as possible with regard to pendulum motion. The result was that the pendulum moved about 10 times as much in the kinetic imagination condition as in the others. The implication of this finding is that the pendulum can be held still and that its movements must be the result of psychological processes. Thus, a second, more comprehensive experiment was designed to isolate strong parametric influences of the pendulum motion.

The results of that experiment (Easton \& Shor, Note 1) indicated that the extent of pendulum motion depended on whether the musculature of the entire arm or just the hand cuffed at the wrist was involved in the suspension of the pendulum. When the arm was freed, the magnitudes of oscillation increased except when subjects were required to concentrate simultaneously on two conceptually antithetical ideas. When subjects directed their attention solely to an image of movement and were not required to perform cognitive tasks with dual components, resultant pendulum oscillations were most extensive, especially when visual and/ or auditory imaginal prompts were present. The auditory prompts consisted of the experimenter speaking a back-and-forth cadence in the harmonic period of the pendulum. The visual prompts consisted of the experimenter moving his hand back and forth beneath the pendulum in its harmonic period. The facilitative effect of the verbal prompts factor proved to be small and was restricted to specific levels of other factors. In sharp contrast, visual prompts exerted an overall facilitative influence, suggesting the potency of visual stimulation on the pendulum effect. It was also found that females tended to create more pendulum movement than males.

The experiment to be presented in this report was designed to make the experimental method more precise and to explore 
ideomotor and visual capture explanations of the pendulum effect. Fulfilling these objectives required automating the means of presenting the visual and auditory imaginal prompts. Visual stimulation was designed which consisted of a vertical white line that oscillated horizontally on a television monitor. Two types of moving auditory stimulation were developed and compared with the visual stimulus. First an ascendingdescending tone (more concrete than the back-and-forth utterances used previously) was developed. The second auditory stimulus was designed with a direct spatial component; a tone appeared to move back and forth through space between the subjects' ears.

Also tested was the effectiveness of the imaginal prompts versus no imaginal prompts under conditions where the subject could either see or not see the actual pendulum motion. A visual capture explanation of the pendulum effect would maintain that sight of the pendulum is crucial, especially when no imaginal prompts are present. If sight was occluded no pendulum movement should result, as the occurrence of small hand movements would be unlikely. Since visual information would be absent, small hand movements in or near the resonant frequency of the pendulum would not only be more easily detected but should not occur at all as they could not result in an image via visual feedback. The ideomotor theory, on the other hand, would not hold that visual feedback of pendulum oscillations is necessary.

\section{Method}

\section{Apparatus and materials}

The pendulum bob consisted of a silver-colored $35-\mathrm{mm}$ film canister, $45 \mathrm{~mm}$ high by $30 \mathrm{~mm}$ in diameter with a tapered top, weighted to $86 \mathrm{~g}$. Attached to the center of its top was a $3 \mathrm{~mm}$ (in diameter) white nylon cord $61 \mathrm{~cm}$ long. When in use the pendulum was held in front of a $90 \mathrm{~cm}$ high by $60 \mathrm{~cm}$ wide black backboard mounted on a $30 \mathrm{~cm}$ high pedestal; the subject stood behind the backdrop, extending the hand holding the pendulum over its top, and looked down at the pendulum against the black top of the pedestal. A removable board was affixed to the top of the backboard which, when in place, occluded sight of the pendulum but not of the visual prompts. A 16-mm movie camera with facility for single-frame time exposures was positioned to take photographs of the backboard area. The white pendulum held in front of the black backboard permitted time exposure photographs of the pendulum's side-to-side motion with excellent contrast and clarity.

Three types of automated imaginal prompts were used. The oscillating visual stimulus was a dc voltage sweep generated by an oscillator, displayed on an oscilloscope, and then videotaped and played on a $53-\mathrm{cm}$ television monitor. The monitor, tipped at a $15^{\circ}$ angle away from a vertical position, was placed $1 \mathrm{~m}$ in front of the subject. The generated vertical white line thus oscillated horizontally on the television screen $25 \mathrm{~cm}$ from side to side. When the occlusion board was not in place the subject could watch the pendulum and at the same time monitor the visual imaginal prompt in attention. The pendulum and television monitor were not spatially interposed. When the board was in place only the monitor was visible.

The first auditory stimulus was an ascendingdescending tone $(450-850 \mathrm{~Hz})$ presented binaurally through earphones. The second auditory stimulus involved the dichotic presentation of two amplitude modulated tones $(500 \mathrm{~Hz})$. The modulation of the tone to one ear was $180^{\circ}$ out of phase with the modulation of the tone to the other ear. The perceived impression was that of a single tone moving back and forth laterally in space, between the ears.

All of the oscillating stimuli were generated sinusoidally and timed to correspond to the exact harmonic period of the pendulum's sinusoidal motion. The phase relationship between the motion of prompts and the pendulum was not controlled in this experiment.

Timers were arranged to provide a $30-\mathrm{sec}$ delay interval after the onset of a trial followed by a 6-sec single-frame time exposure. The 30sec delay interval allowed time for the pendulum to overcome inertia and build up to a stable level of motion. The 6-sec time exposure allowed the pendulum to sweep out about four cycles of oscillation. As hand movements were uniformly very small during the trial, the picture on the photographic negative consisted of a shaded isosceles triangle with a curved base, representing the area of the pendulum's motion. Protractor measurements of the angle of the apex of the triangle were taken directly from an enlarged image of the negative frame. Inter- and intrajudge differences were found to be random and within $.2^{\circ}$ yielding reliability coefficients of .95 and above.

Subjects were required to wear a heavy leather glove lined with cotton. This was sufficient to sharply reduce or to remove reported awareness of tactual feedback.

\section{Subjects}

Eight male and seven female introductory psychology students fulfilling requirements at the 
University of New Hampshire served as subjects.

\section{Procedure}

Subjects were run individually. Upon entering the experimental room subjects were told that the experimenter was interested in studying imagination and concentration. They were informed that their basic task was simply to hold the pendulum between thumb and forefinger over the backboard, and while keeping their eyes on the pendulum, to imagine that the pendulum moves back and forth. The subjects were asked to refrain from moving the pendulum deliberately. They were told to keep their head and body relaxed but still. They were asked, as far as their basic task was concerned, to try to exclude other thoughts from active processing. The experimenter attempted to maintain a neutral, detached attitude while conducting the experimental session.

Further instructions to subjects indicated that on some trials auxiliary stimuli would be presented in either an auditory or visual mode that could potentially strengthen their imaginal efforts. They were asked to incorporate this information into their imaginal constructions to the extent they found it helpful.

About $5 \mathrm{~min}$ were then spent in administering a standard set of practice trials to provide warmup and allow the subjects to become familiar with the general task.

The experimental design consisted of the factorial combination of three within-subjects factors: a four-level type-of-imaginal-prompt factor (no imaginal prompt, ascending-descending tone, spatial tone, visual prompt), a two-level occlusion factor (pendulum oscillation not visible, pendulum oscillations visible), and a three-level blocks-oftrials factor (first, second, and third block of trials). Three blocks of eight trials each were run with the order of trials within each block

TABLE 1

Means of Maximum Angle of Pendular Motion for all Treatment Combinations

\begin{tabular}{|c|c|c|c|c|}
\hline Block & None & $\begin{array}{l}\text { Stim } \\
\text { Ascending } \\
\text { descending } \\
\text { tone }\end{array}$ & $\begin{array}{l}\text { Ius } \\
\text { Spatial } \\
\text { tone }\end{array}$ & $\begin{array}{l}\text { Visual } \\
\text { spatial }\end{array}$ \\
\hline \multicolumn{5}{|c|}{ Pendulum not visible } \\
\hline $\begin{array}{l}1 \\
2 \\
3\end{array}$ & $\begin{array}{l}2.5 \\
1.9 \\
2.5\end{array}$ & $\begin{array}{l}3.1 \\
2.7 \\
3.3\end{array}$ & $\begin{array}{l}2.7 \\
3.3 \\
3.7\end{array}$ & $\begin{array}{l}4.7 \\
4.7 \\
4.8\end{array}$ \\
\hline
\end{tabular}

Pendulum visible

\begin{tabular}{lllll}
\hline 1 & 4.0 & 4.7 & 4.9 & 6.1 \\
2 & 4.1 & 5.3 & 5.5 & 6.5 \\
3 & 4.3 & 6.0 & 6.3 & 6.7 \\
\hline
\end{tabular}

$N=15$. Entries are in degrees. randomly determined. The eight trials per block represented the crossed levels of the type-ofimaginal-prompt and occlusion factors.

\section{Results}

The data were analyzed by means of a three-factor analysis of variance with repeated measures. No sex differences (main or interactive) emerged in an initial fourfactor analysis. Means for all experimental conditions are presented in Table 1. A graphic depiction of the significant effects is presented in Figure 1. As can be seen, the magnitude of pendulum movement was significantly larger when sight of the actual pendulum oscillations was permitted, $F(1$, 14) $=6.09, p<.05$. All three types of imaginal prompts significantly facilitated the buildup of pendulum motion, $F(3,42)=$ $18.55, p<.001$. However, a NewmanKeuls comparison indicated that the presence of the visual stimulus resulted in significantly larger swings $(p s<.01)$ than either of the auditory stimuli. The latter two stimuli did not differ from one another but were each significantly different from the no-imaginal-prompt condition ( $p s<$ $.01)$. In contrast to this objective finding, postexperimental questioning of the subjects revealed that 8 of the 15 regarded the spatial tone as the most subjectively compelling and suggestive of movement. The pattern of results that emerged from an analysis of just these 8 subjects was not appreciably different from that of the main analysis. Thus, the spatial component added to the auditory stimulus was sufficient to strengthen conscious introspective imagination but did not produce as much objective effect as a visual-spatial imaginal prompt.

The lack of any interaction among the three factors in this experiment, $F(6,84)$ $=.15$, is a noteworthy finding. It indicates that the effect of imaginal prompts was significant both when the pendulum was visible and not visible. Moreover, the rank order of the facilitative effects of the different prompts remained constant with and without visual feedback.

The blocks-of-trials factor was not significant and did not interact with other factors. 


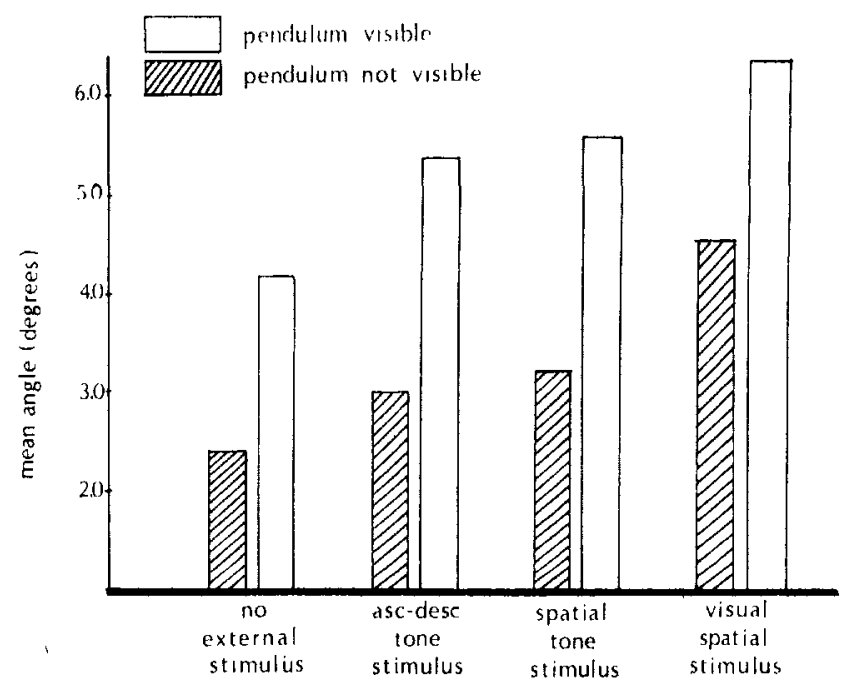

FIGURE 1. The effect of imaginal prompts with and without visual feedback of pendulum oscillations. (Asc $=$ ascending, desc $=$ descending.)

\section{Discussion}

It appears relevant to comment on the subjective quality of the illusory effect of the pendulum's motion. The illusion involves the subjects' failure to notice the small muscle movements of the arm and hand that set the pendulum into oscillation. The relatively poor monitoring of kinesthetic information could be the consequence of an ideomotor process in which muscle movements automatically occur as covert accompaniments of a central nervous system activity. On the other hand, the failure to detect muscle movements could occur because simultaneous visual information (sight of pendulum oscillations) dominates conscious processing. In this case, hand movements could be generated incipiently, resulting in a constructed image of movement via visual feedback of pendulum motion. The results from the present study would seem to indicate that both central and peripheral processing are operating.

When actual pendulum oscillations are visible, the magnitude of pendulum movement is larger, presumably because sight of pendulum motion easily and directly fulfills the instructions to construct an imagination of movement. Further, the phenomenon of visual capture suggests that larger arm and hand movements could go undetected under such conditions. This line of reasoning would not seem to apply when sight of the pendulum is prevented, however. Visual capture could not exist and no amount of actual pendulum movement could fulfill the instruction to create an image of movement. Yet, the pendulum moves when sight of actual movement is prevented, albeit in an attentuated form. In spite of the attentuation, the relation among the imaginal prompts prevails and is significant. A reasonable interpretation is that the attenuated movement occurs in an ideomotor way.

One might argue that the imaginal prompts that were present when the pendulum was not visible constituted sufficient information for intersensory capture to occur. Even though the phase relation between the motion of the pendulum and prompts was not controlled, the perception of controlled frequency, sinusoidal movement could dominate conscious processing. This indeed may be true and it should caution one against a hasty conclusion that the differences in Figure 1 between movement of the pendulum with and without sight of pendular oscillations represents the contribution of visual capture as an independent influence beyond ideomotor behavior. Be that as it may, the pendulum did move when sight of the actual pendulum 
motion was occluded and when no imaginal prompts were present. That specific condition may represent pure ideomotor behavior.

Consistent with the above discussion is the proposal that the facilitative effects of the imaginal prompts are the restult of visual capture and the incorporation of stimulus information into imaginal processing. It is known that vision strongly dominates conscious processing while audition does not (Pick et al., 1969). The present finding of a greater facilitative effect for the visual prompt supports this interpretation.

One final point is worth noting. The ordinal relation among the imaginal prompts remained the same with and withotit visual feedback. Since the subject also wore a heavy glove, it seems unlikely that sufficient conscious information existed in the absence of visual and tactual feedback to allow the deliberate production of the obtained small, precisely ordered magnitudes of pendulum motion. It would therefore be difficult to attribute the results solely to "good subject roles" (Orne, 1969).

The general conclusion to be drawn from the present findings is that the Chevreul pendulum effect, though historically considered only as a standard test of "suggestibility," is a reliable experiment phenomenon. It appears to be related to ideomotor theory, visual capture, and the incorporation of stimulus information into imagination.

\section{REFERENCE NOTE}

1. Easton, R. D., \& Shor, R. E. An experimental analysis of the Cheoreul pendulum illusion. Paper presented at the 24th annual convention of the Society for Clinical and Experimental Hypnosis, Boston, October 1972.

\section{REFERENCES}

Chevreul, M. E. Lettre à M. Ampère sur une classe particulaires. Review des deux mondes (2nd series), 1833, 2, 258-266.

Chevreul, M. E. De la baquetta divinatoire, du pendulue explorateur, des tables tournates, an point de vue de l'histoire de la critique et de la méthode éxpérimentale. Paris: Mallet-Bachelier, 1854.

Crandall, S. H., \& Marks, W. D. Random vibration in mechanical systems. New York: Academic Press, 1963.
Gibson, J. J. Adaptation, after-effect, and contrast in the perception of curved lines. Journal of Experimental Psychology, 1933, 16, 1-31.

Greenwald, $A . G$., Sensory feedback mechanisms in performance control: With special reference to the ideo-motor mechanism. Psychological Review, 1970, 77, 73-99.

Greenwald, A. G. On doing two things at once: Time sharing as a function of ideomotor compatibility. Journal of Experimental Psychology, $1972,94,52-57$.

Greenwald, A. G., \& Shulman, H. G. On doing two things at once: II. Elimination of the psychological refractory period effect. Journal of Experimental Psychology, 1973, 101, 70-76.

James, W. The principles of psychology (2 vols.). New York: Dover, 1950. (Originally published, 1890.)

McGuigan, F. J., \& Schoonover, R. A. (Eds.). The psychophysiology of thinking: Studies of covert processes. New York: Academic Press, 1973.

Miller, N. E. Learning of visceral and glandular responses. Science, 1969, 163, 434-445.

Nielsen, T. I. Volition: A new experimental approach. Scandinavian Journal of Psychology, 1963, 4, 225-230.

Orne, M. T. Demand characteristics and the concept of quasi-controls. In R. Rosenthal \& R. Rosnow (Eds.), Artifact in behavioral research. New York: Academic Press, 1969.

Perky, C. W. An experimental study of imagination. American Journal of Psychology, 1910, $21,422-452$.

Pick, H. L., Warren, D. H., \& Hay, J. C. Sensory conflicts in judgments of spatial direction. Perception \& Psychophysics, 1969, 6, 203-205.

Rock, I. The nature of perceptual adaptation. New York: Basic Books, 1966.

Ryan, T. A. Interrelations of the sensory systems in perception. Psychological Bulletin, 1940, 37, 659-698.

Segal, S. J. Assimilation of a stimulus in the construction of an image: The Perky effect revisited. In $P$. Sheehan (Ed.), The function and nature of imagery. New York: Academic Press, 1972.

Sheehan, P. W. The function and nature of imagery. New York: Academic Press, 1972.

Smith, K. Curare drugs and total paralysis. Psychological Reviewe, 1964, 71, 77-79.

Smith, K. Behavior and conscious experience: $A$ conceptual analysis. Athens: Ohio University Press, 1969.

Smith, S. M., Brown, H. O., Teman, J. E. P., \& Goodman, L. S. The lack of cerebral effects of d-tubocurarine. Anesthesiology, 1947, 8, 1-14.

Thorndike, E. L. Ideo-motor action. Psychological Revierw, 1913, 20, 91-106.

Watson, J. B. Behaviorism (2nd ed.). New York: Norton, 1930.

(Received December 20, 1974) 